\title{
Influenza vaccination to Reduce Cardiovascular Events : Time to Act
}

\author{
Abdullah Al Shafi Majumder \\ Professor of Cardiology \& Ex-Director, National Institute of Cardiovascular Diseases, Dhaka.
}

(Cardiovasc. j. 2017; 10(1): 1-2)

Evidence from different studies suggest that there is relation with influenza infection and acute and chronic cardiovascular events. ${ }^{1,2}$ There is increased incidence of acute myocardial infarction with increased incidence of influenza infection. An author finds 2 times more incidence of acute myocardial infarction in seasons with influenza epidemic. ${ }^{3}$ As well there is increased incidence of worsening of heart failure with more hospitalisation during increased incidence of influenza. ${ }^{4}$ The pathophysiology linking the influenza infection with these events is not difficult to understand. As with any infection, influenza infection leads to increased metabolic demand, increased adrenaline level and there develops hypercoagulable state. There is also endothelial dysfunction and inflammatory reaction. These factors facilitate the rupture of atherosclerotic plaque of the coronary arteries leading to acute coronary syndrome. ${ }^{5}$

In other aspect influenza infection worsens heart failure necessitating more hospitalisation. Influenza infection directly depresses the myocardial function - myocarditis and myocyte necrosis ; through proinflammatory cytokines there is depression of myocardial function ; increased sympathetic stimulation results in increased oxygen demand on already failing heart. ${ }^{5}$

Arguments based on pathophysiology are clinically validated on a number of observational studies, metaanalyses and experts opinion. After the validation next step is to prevent this - simple logic!

It has been accepted that vaccination against influenza infection is quite effective to attenuate the adverse effects. Thus providing vaccination to susceptible persons is supposed to decrease the cardiovascular events. There are number of trials to show the beneficial effects of vaccination on cardiovascular events. ${ }^{6,7}$

Among many studies, one recent brilliant study from University of Oxford deserves more attention which tries to investigate impact of influenza vaccination on the risk of hospitalisation. ${ }^{4}$ They analysed more than 59.000 patients over more than 2 decades. They compared hospitalisation rate (due to cardiovascular, respiratory and all-cause ) on an individual patient level for consecutive 2 years : one year the patient was vaccinated and the other year not vaccinated. They found lower risk for cardiovascular hospitalisation including hospitalisation for heart failure during vaccinated year compared with the risk in unvaccinated year.

Though there is good evidence to support the use of vaccination in high risk patients, there is less use of it than it should be. ${ }^{1}$ In one trial its use was as low as $21 \%$ in the recommended group. WHO recommends annual influenza vaccination in the high risk group patients that is patients with cardiac diseases -atherosclerotic heart disease, cardiomyopathy/ chronic congestive heart failure and congenital heart disease. In patients with established cardiovascular disease, evidence suggests that influenza vaccination may reduce cardiovascular mortality and combined cardiovascular event.1,3,6 ESC guideline recommends 8,9 "Annual influenza vaccination may be considered in patients with estabileshed CVD “ It is Class IIb with level of evidence C. In future large-scale and adequately powered studies targeting the effectiveness and safety of anti-influenza vaccination may assess beyond any doubt the costeffectiveness of anti-influenza vaccination in $\mathrm{HF}$ patients (secondary prevention) or those at increased

Address of Correspondence : Abdullah Al Shafi Majumder, Professor of Cardiology \& Ex-Director, National Institute of Cardiovascular Diseases, Dhaka. 
risk for HF development (primary prevention). One may expect that the data that are accumulating will make recommendation Class I in the next guideline.

Before end, I may quote Vardney and Solomon of Harvard Medical School of USA regarding influenza vaccination to reduce cardiovascular events : "There are few interventions in all of Medicine that are as low-cost, low-risk, well tolerated or easy to administer and with such large clinical benefits. Don't we owe it to our patients to offer them this one-shot deal? "5

\section{References: :}

1. Clar C, Oseni Z, Flowers N, Keshtkar-Jahromi M, Rees $\mathrm{K}$ Influenza vaccines for preventing cardiovascular disease (Review). Cochrane Database of Systematic Reviews 2015, Issue 5. Art. No.: CD005050

2. Shrinivas Hebsur, Erik Vakil, William J. Oetgen, Princy N. Kumar, Daisy F. Lazarous, Influenza and Coronary Artery Disease: Exploring a Clinical Association With Myocardial Infarction and Analyzing the Utility of Vaccination in Prevention of Myocardial Infarction.Rev Cardiovasc Med. 2014;15(2):168-175

3. C Raina MacIntyre, Anita E Heywood, Pramesh Kovoor, Iman Ridda, Holly Seale, Timothy Tan, Zhanhai Gao, Anthea L Katelaris, Ho Wai Derrick Siu, Vincent Lo, Richard Lindley,Dominic E Dwyer. Ischaemic heart disease, influenza and influenza vaccination: a prospective case control study. Heart 2013;99: 1843-1848

4. Mohseni H, Kiran A, Khorshidi R, Rahimi K. Infuenza vaccination and risk of hospitalisation in patients with heart failure : a self-controlled case series study. Eur Hear J 2017;38: 326-333
5. Orly Vardeny and Scott D. Solomon. Influenza vaccination : a one- shot deal to reduce cardiovascular events. Eur Heart J 2017;38:334-337

6. Nikolaos P. E. Kadoglou, Frank Bracke, Tim Simmers, Sotirios Tsiodras, John Parissis. Influenza infection and heart failure-vaccination may change heart failure prognosis. Heart Fail Rev 2017; 22:329-336

7. C Raina MacIntyre, Abela Mahimbo, Aye M Moa, Michelle Barnes. Influenza vaccine as a coronary intervention for prevention of myocardial infarction. Heart 2016;102:1953-1956.

8 Massimo F. Piepoli, ArnoW. Hoes, Stefan Agewall, Christian Albus , Carlos Brotons, Alberico L. Catapano, Marie-Therese Cooney, Ugo Corra', Bernard Cosyns , Christi Deaton, Ian Graham, Michael Stephen Hall, F. D. Richard Hobbs , Maja-Lisa Løchen , Herbert Lo“ llgen , Pedro Marques-Vidal, Joep Perk, Eva Prescott, Josep Redon, Dimitrios J. Richter, Naveed Sattar, Yvo Smulders, Monica Tiberi, H. Bart van derWorp, Ineke van Dis, W. M. Monique Verschuren . 2016 European Guidelines on the prevention of cardiovascular diseases in clinical practice. Eur Heart J doi:10.1093/eurheartj/ ehw106 2016;22

9 Piotr Ponikowski, Adriaan A. Voors, Stefan D. Anker, He'ctor Bueno, John G. F. Cleland, Andrew J. S. Coats, Volkmar Falk, Jose' Ramón Gonza'lezJuanatey, Veli-Pekka Harjola, Ewa A. Jankowska , Mariell Jessup, Cecilia Linde, Petros Nihoyannopoulos, John T. Parissis, Burkert Pieske, Jillian P. Riley, Giuseppe M. C. Rosano, Luis M. Ruilope, Frank Ruschitzka, Frans H. Rutten, Peter van der Meer. 2016 ESC Guidelines for the diagnosis and treatment of acute and chronic heart failure. Eur Heart J 2016;37:2129-2200. 